\title{
Effect of Physical Fields on Highly Resinous Oil
}

\author{
Iulia V. Loskutova, \\ Anastasia V. Morozova and Galina I. Volkova* \\ Institute of Petroleum Chemistry SB RAS \\ Tomsk, Russian Federation
}

Received 14.03.2021, received in revised form 12.04.2021, accepted 23.05.2021

\begin{abstract}
This paper studies the effect of physical fields (i.e. ultrasonic and constant magnetic fields) on structural and energy properties (viscosity, limiting shear stress, pour point, activation energy of viscous flow) as well as kinetic (antioxidant) properties of highly resinous problematic oil. The results were obtained via viscometry, determining of pour point and voltammetric method of oxygen electroreduction. It was shown that the treatment of studied oil by acoustic and magnetic fields lead to decrease in viscosity and temperature parameters. Combined treatment displays an additional reduction of viscosity and pour point.
\end{abstract}

Keywords: ultrasonic treatment, magnetic treatment, highly resinous oil, antioxidant activity.

Citation: Loskutova Iu.V., Morozova A.V., Volkova G.I. Effect of physical fields on highly resinous oil, J. Sib. Fed. Univ. Chem., 2021, 14(2), 226-233. DOI: 10.17516/1998-2836-0231

(C) Siberian Federal University. All rights reserved

This work is licensed under a Creative Commons Attribution-NonCommercial 4.0 International License (CC BY-NC 4.0).

* Corresponding author E-mail address: reoloil@ipc.tsc.ru 


\title{
Воздействие физических полей \\ на высокосмолистую нефть
}

\author{
Ю. В. Лоскутова, А. В. Морозова, Г.И. Волкова \\ Институт химии нефти СО РАН \\ Российская Федерации, Томск
}

\begin{abstract}
Аннотация. Изучено влияние физических полей (ультразвукового и постоянного магнитного) на структурно-энергетические (вязкость, предельное напряжение сдвига, температуру застывания, энергию активации вязкого течения) и кинетические (антиоксидантные) свойства проблемной высоковязкой нефти. Результаты были получены с помощью метода вискозиметрии, определения температуры застывания, вольтамперометрического метода электровосстановления кислорода. Показано, что воздействие на исследуемую нефть как акустическим, так и магнитным полем приводит к понижению вязкостно-температурных параметров. Сочетание воздействий сопровождается дополнительным понижением вязкости и температуры застывания нефти.
\end{abstract}

Ключевые слова: ультразвуковое воздействие, магнитная обработка, высокосмолистая нефть, антиоксидантная активность.

Цитирование: Лоскутова, Ю. В. Воздействие физических полей на высокосмолистую нефть / Ю. В. Лоскутова, А. В. Морозова, Г. И. Волкова // Журн. Сиб. федер. ун-та. Химия, 2021, 14(2). С. 226-233. DOI: 10.17516/1998-2836-0231

\section{Введение}

За последние десятилетия все более широкое применение на нефтепромысле находят экономичные и экологически безопасные технологии обработки добываемой нефти физическими полями для предотвращения и удаления нефтяных отложений в стволе скважины, на нефтяном оборудовании и в призабойной зоне [1]. Волновые методы обработки внедряются также для увеличения добычи тяжелых нефтей, улучшения качества нефти [2-4].

Применение комплексных методов подготовки нефти к транспорту с использованием комбинирования различных видов внешних воздействий является одним из приоритетных направлений развития нефтедобычи, позволяющих существенно сократить экономические потери и улучшить экологическую ситуацию на промысле.

На примере высокосмолистой проблемной нефти было изучено влияние ультразвуковой и магнитной обработки, а также сочетание воздействий на структурно-энергетические и кинетические характеристики.

\section{Объекты и методы исследования}

В работе воздействие ультразвуковой обработки (УЗО) и магнитной обработки (МО) на высокосмолистую тяжелую нефть Боровского месторождения (Самарская область), содержащую порядка 7 \% мас. асфальтенов, 16 \% мас. смол и 4 \% мас. парафинов, изучали по изменению температуры застывания Тз на приборе ИНПН «Кристалл» (Россия), предельного напряжения

$$
-227-
$$


сдвига $\tau_{0}$, динамической вязкости $\eta$ при $20^{\circ} \mathrm{C}$ и энергии активации вязкого течения Еа(вт) в температурном интервале от 15 до минус $20^{\circ} \mathrm{C}$ на ротационном вискозиметре Brookfield LVDV III Ultra (USA).

В работе окислительно-восстановительную активность нефти оценивали с помощью вольтамперометрического метода катодного восстановления кислорода на установке с использованием ртутного пленочного электрода [5]. Суть определения АОА заключается в регистрации зависимости тока, протекающего в цепи электрохимической ячейки, от приложенного к ее электродам напряжения. Аналитическим сигналом является максимальное значение тока электровосстановления кислорода (ЭВ $\mathrm{O}_{2}$ ) в присутствии АО, содержащихся в исследуемом образце. Концентрацию анализируемого вещества (нефти) в растворе фонового электролита варьировали от 0,05 до 0,3 г/л.

Обработку исследуемой нефти магнитным полем осуществляли с помощью магнитной системы активатора проточного типа серии МАУТ (ООО ПКФ «Экси-Кей», г. Томск), созданного на основе постоянных магнитов Ne-Fe-B (индукция 0,3-0,4 T) (рис. 1). Обработка проводилась при комнатной температуре в проточном режиме со скоростью $10 \mathrm{~cm}^{3} / \mathrm{Mин}$.

Ультразвуковую обработку нефти проводили с использованием ультразвукового дезинтегратора Ultrasonic generator TS-4000М (РФ) при интенсивности акустического поля $10 \mathrm{BT} / \mathrm{cm}^{2}$ и частоте поля 22 кГц (рис. 2).

Установка состоит из генератора с высокой мощностью и интенсивностью ультразвуковых колебаний. Работа генератора основана на преобразовании трехфазного электрического напряжения питающей сети в однофазное напряжение ультразвуковой частоты.

\section{Обсуждение результатов}

Результаты исследований реологического поведения нефти Боровского месторождения до физического воздействия, после 1- и 3-минутной УЗО, после МО и последовательной обра-

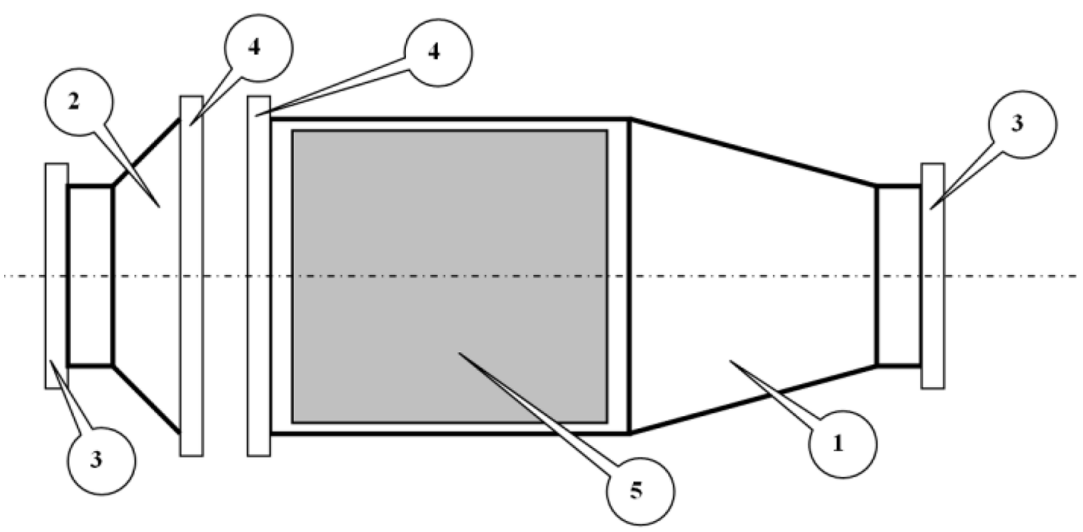

Рис. 1. Схема устройства магнитоактиватора МАУТ: 1 - несущий корпус; 2 - крышка корпуса; 3 торцевые фланцы для крепления устройства на трубопроводе; 4 - торцевые фланцы для соединения корпуса и крышки устройства; 5 - магнитная система устройства

Fig. 1. Diagram of MAUT magnetoactivator device: 1 - carrying body; 2 - housing cover; 3 - end flanges for fastening the device to the pipeline; 4 - end flanges for connecting the body and the cover of the device; 5 magnetic system of the device 


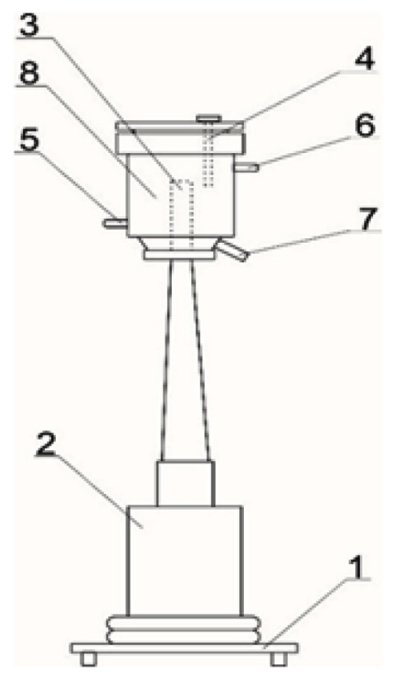

Рис. 2. Схема ультразвукового дезинтегратора Ultrasonic generator TS-4000M: 1 - основание; 2 магнитострикционный преобразователь; 3 - рабочий наконечник; 4 - термопара; 5 - штуцер (вход); 6 штуцер (выход); 7 - штуцер для слива образца; 8 - реактор

Fig. 2. Diagram of the ultrasonic disintegrator Ultrasonic generator TS-4000M: 1 - base; 2 - magnetostrictive transducer; 3 - working tip; 4 - thermocouple; 5 - fitting (inlet); 6 - fitting (outlet); 7 - fitting for draining the sample; 8 - reactor

ботки физическими полями при $20^{\circ} \mathrm{C}$ изображены в виде кривых зависимости динамической вязкости нефти Боровского месторождения от скорости сдвига (рис. 3).

Лишь на начальном участке сдвиговых скоростей до 5-8 $\mathrm{c}^{-1}$ нефть до и после обработки обладает ярко выраженной структурной вязкостью. Далее с увеличением сдвиговой скорости значения на кривой выходят на линейное плато, что соответствует эффективной вязкости нефти с разрушенной структурой.

На рис. 4 представлено, как влияют условия обработки на значения предельного напряжения сдвига $\tau_{0}$ и эффективной вязкости $\mu$. Эти величины очень важны для характеристики течения нефти в трубопроводе. Очевидно, что именно после совместной обработки (УЗО+МО) необходимо приложить минимальное давление для перехода обработанной нефти из состояния покоя в состояние течения (рис. 4а) и для сохранения минимальной вязкости в трубопроводе (рис. 4б).

Особенности процессов структурообразования нефтяных систем при физических воздействиях изучали на примере изменения величины энергии активации вязкого течения $\mathrm{E}_{\text {a(вт)}}$, которую рассчитывали по уравнению Френкеля-Эйринга (1):

$$
\eta=A \exp ^{\mathrm{Ea}(\mathrm{Br}) / \mathrm{RT}},
$$

где $\eta$ - динамическая вязкость; А - предэкспоненциальный коэффициент, включающий в скрытом виде зависимость вязкости от других параметров структуры, в частности молекулярной массы; $\mathrm{E}_{\mathrm{a(sт)}}$ - энергетический барьер процесса флуктуационного перехода (энергия активации процесса вязкого течения); $\mathrm{R}$ - универсальная газовая постоянная; $\mathrm{T}$ - абсолютная температура [5]. 


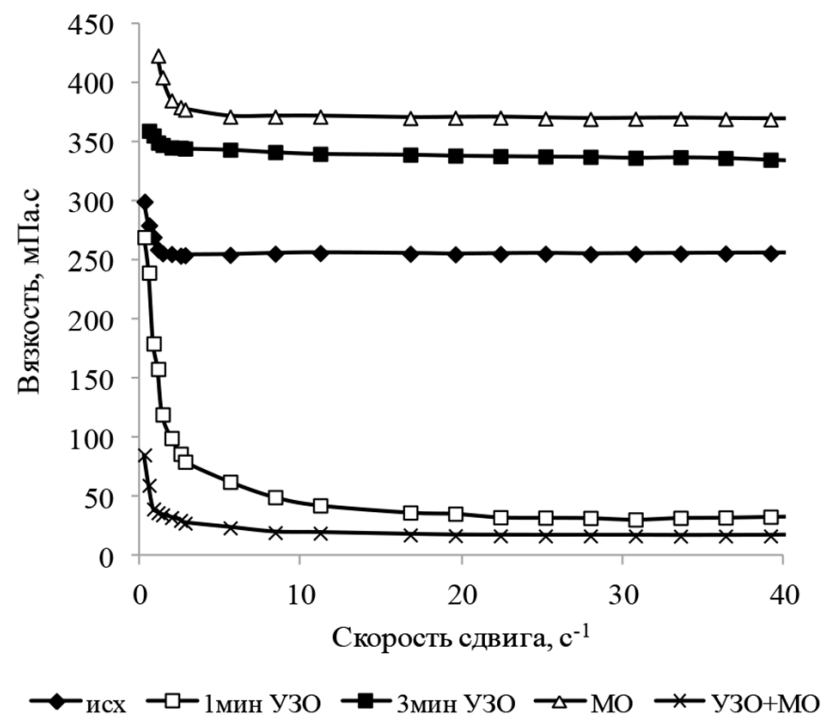

Рис. 3. Кривые зависимости динамической вязкости нефти Боровского месторождения от скорости сдвига после 1 и 3 мин УЗО, после МО и совместной УЗО и МО

Fig. 3. Dependence of the dynamic viscosity of oil from Borovskoye field on the shear rate after 1 and 3 minutes of UST, after MT, and after UST+MT complex treatment

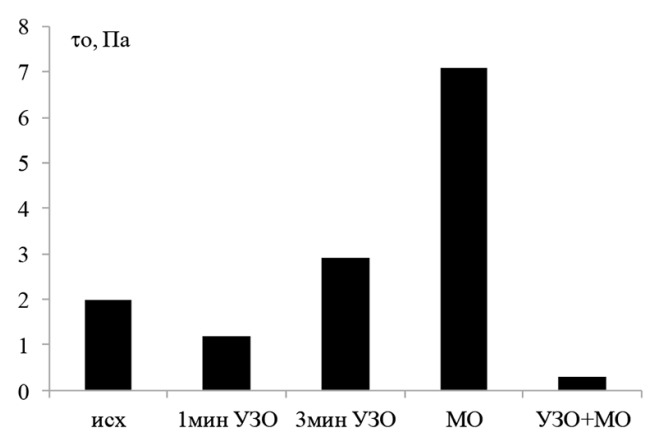

a

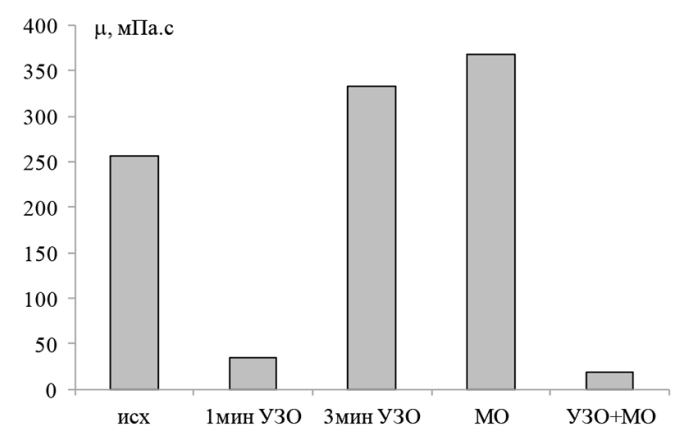

6

Рис. 4. Влияние параметров физической обработки на значения (а) предельного напряжения сдвига $\tau 0$ и (б) эффективной вязкости $\mu$ при скорости сдвига $56 \mathrm{c}^{-1}$

Fig. 4. Influence of physical treatment parameters on the values of (a) limiting shear stress $\tau 0$ and (б) effective viscosity $\mu$ at a shear rate of $56 \mathrm{~s}^{-1}$

В табл. 1 приведены значения температуры застывания Тз, предельного напряжения сдвига $\tau_{0}$ и динамической вязкости $\eta$ при $20^{\circ} \mathrm{C}$, а также энергии активации вязкого течения $\mathrm{E}_{\mathrm{a}(\mathrm{вт)})}$

Воздействие на исследуемую нефть акустическим полем приводит к снижению Тз на $20,6{ }^{\circ} \mathrm{C}, \tau_{0}$ на 40 \% и $\eta$ на 87 \%. С понижением температуры эффективность УЗО падает, а величина $\mathrm{E}_{\mathrm{a(в)})}$, характеризующая прочность связей в нефтяных ассоциатах в каждом структурном состоянии, возрастает. Обработка магнитным полем снижает Тз лишь на $12,7^{\circ} \mathrm{C}$, а величина $\eta$ и $\tau_{0}$, напротив, возрастает на 30 \% и в 1,6 раза соответственно. При комплексном 
Таблица 1. Влияние физической обработки на реологические характеристики нефти

Table 1. Effect of physical treatment on the rheological characteristics of oil

\begin{tabular}{|c|c|c|c|c|}
\hline Образец нефти & $\mathrm{T}_{3}{ }^{\circ} \mathrm{C}$ & $\tau_{0}{ }^{20}$, Па & $\eta^{20}, \mathrm{мПа \cdot с}$ & $\mathrm{E}_{\text {а(вт), }}$ кДж/моль \\
\hline До обработки & $-20,2$ & 2,0 & 256,5 & 29,4 \\
\hline УЗО & $-40,8$ & 1,2 & 34,2 & 30,6 \\
\hline МО & $-32,9$ & 2,9 & 332,6 & 29,5 \\
\hline МО $\rightarrow$ УЗО & $-43,4$ & 7,1 & 367,8 & 30,7 \\
\hline УЗО $\rightarrow$ МО & $-35,3$ & 0,3 & 18,2 & 32,9 \\
\hline
\end{tabular}

воздействии на магнитообработанную нефть УЗ полем $(\mathrm{MO} \rightarrow \mathrm{У3O})$ Тз максимально снижается на $23,2^{\circ} \mathrm{C}, \tau_{0}$ и вязкость возрастают. После магнитного воздействия на активированную ультазвуком нефть (УЗО $\rightarrow \mathrm{MO})$ наблюдается максимальный эффект снижения $\tau_{0}$ и $\eta$ в 6,7 и 14,1 раза соответственно.

Окислительно-восстановительную активность нефти до и после воздействия физическими полями оценивали с помощью вольтамперометрического метода катодного восстановления кислорода на установке с использованием ртутного пленочного электрода [5]. При добавлении содержащего антиоксиданты (АО) образца нефти в фоновый электролит происходит ингибирование процесса восстановления кислорода. Система детектирования основана на определении по вольтамперограмме степени ингибирования предельного тока кислорода. Генерация активных кислородных радикалов происходит на поверхности рабочего электрода по схеме (2-4):

$$
\begin{gathered}
k \\
\mathrm{O}_{2}+\overline{\mathrm{e}} \rightleftarrows \mathrm{O}_{2}{ }^{-}, \\
\mathrm{O}_{2}{ }^{--}+\mathrm{H}^{+} \rightleftarrows \mathrm{HO}_{2}, \\
\mathrm{HO}_{2}{ }^{-}+\mathrm{H}^{+}+\overline{\mathrm{e}} \rightleftarrows \mathrm{H}_{2} \mathrm{O}_{2} .
\end{gathered}
$$

В качестве фонового электролита (ФЭ), в котором растворяли навеску анализируемого вещества, использовали раствор $0,1 \mathrm{M} \mathrm{NaClO} 4$ в смеси толуол:этанол (1:1). Концентрацию анализируемого вещества в растворе варьировали от 0,05 до 0,25 г/л (ppm).

Строили график зависимости функции $\left(1-\mathrm{I}_{0}\right)$ от времени $t$ протекания реакции восстановления кислорода в присутствии образца в ФЭ, после обработки которого методом наименьших квадратов рассчитывали тангенс угла наклона по линейной части кривой $\mathrm{d}(1-\mathrm{I} / \mathrm{I} 0) / \mathrm{d} t$ и определяли кинетический критерий антиокислительной активности $K$ (мкмоль/(л·мин)) по формуле (5):

$$
K=\mathrm{C}^{0}{ }_{\mathrm{O} 2} \cdot\left(1-\mathrm{I} / \mathrm{I}_{0}\right) / t
$$

где I - ток электровосстановления (ЭВ) $\mathrm{O}_{2}$ в присутствии $\mathrm{AO}$ в растворе, мкА; $\mathrm{I}_{0}$ - ток ЭВ $\mathrm{O}_{2}$ в отсутствие $\mathrm{AO}$ в растворе, мкА; $\mathrm{C}_{\mathrm{O}}^{0}-$ исходная концентрация $\mathrm{O}_{2}$ в растворе, мкмоль/л; $t-$ время протекания реакции взаимодействия АО с активными кислородными радикалами, мин.

$$
-231-
$$




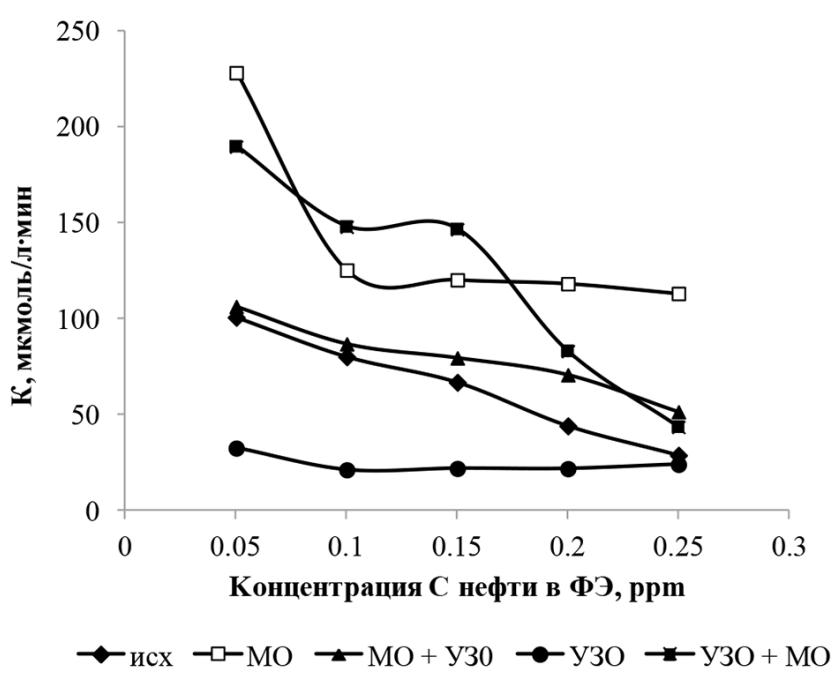

Рис. 5. Зависимость кинетического критерия $K$ антиоксидантной активности от концентрации $C$ нефти в фоновом электролите

Fig. 5. Dependence of the kinetic criterion $K$ of antioxidant activity on the concentration $C$ of oil in the background electrolyte

На рис. 5 представлена зависимость кинетического критерия $K$ антиоксидантной активности нефти Боровского месторождения от концентрации С нефти в ФЭ для различных условий обработки физическими полями.

Характер изменения величины кинетического критерия $K$ для всех образцов нефти в зависимости от концентрации навески С в ФЭ существенно различается. Так УЗО высокосмолистой нефти приводит к резкому снижению величины $K$, а обработка магнитным полем, напротив, к его существенному росту. Высокие значения коэффициента $K$ наблюдаются после комплексной обработки нефти в последовательности УЗО $\rightarrow \mathrm{MO}$. С увеличением концентрации С в ФЭ антиоксидантная активность нефти, кроме магнитного воздействия, снижается до близких величин.

\section{Заключение}

Воздействие на тяжелую высокосмолистую нефть Боровского месторождения акустическим полем приводит к снижению ее реологических характеристик и температуры застывания на $20^{\circ} \mathrm{C}$. С понижением температуры эффективность УЗО падает, а величина энергии активации вязкого течения, напротив, возрастает. В результате обработки нефти магнитным полем наблюдается снижение температуры застывания при росте вязкостных характеристик. При комплексном воздействии на магнитообработанную нефть УЗ-полем (МО $\rightarrow$ УЗ) Тз максимально снижается на $23,2{ }^{\circ} \mathrm{C}$, а после магнитного воздействия на активированную ультразвуком нефть $(\mathrm{У} О \rightarrow \mathrm{MO})$ наблюдается максимальный эффект снижения предельного напряжения сдвига и вязкости.

Содержащиеся в нефти природные ПАВ, смолы и асфальтены, обладают антиокислительной активностью за счет наличия в них функциональных групп, имеющих подвижный атом 
водорода или азота. Изменение антиокислительной активности и вязкостно-температурных свойств исследуемой нефти после различного вида воздействия физическими полями свидетельствует о протекании в них различного рода ассоциативных превращений.

\section{Благодарности / Acknowledgments}

Работа выполнена в рамках государственного задания ИХН СО РАН, финансируемого Министерством науки и высшего образования Российской Федерации.

This work was supported by the Ministry of Science and Higher Education of the Russian Federation.

\section{Список литературы / References}

1. Волкова Г.И., Лоскутова Ю.В., Прозорова И.В., Березина Е.М. Подготовка и транспорт проблемных нефтей. Томск: Из-во ТГУ, 2015. 136 с. [Volkova G.I., Loskutova Iu.V., Prozorova I.V., Berezina E.M. Treatment and transportation of problem oils. Tomsk: Publishing TGU, 2015. 136 p. (In Russ.)]

2. Salehzadeh M., Akherati A., Ameli F., Dabir B. Experimental Study of Ultrasonic Radiation on Growth Kinetic of Asphaltene Aggregation and Deposition. The Canadian Journal Of Chemical Engineering, 2016. Vol. 94. P. 2202-2209. https://doi.org/10.1002/cjce.22593.

3. Tao R., Xu X. Reducing the Viscosity of Crude Oil by Pulsed Electric or Magnetic Field. Energy\&Fuels, 2006. Vol. 20(5). P. 2046-2051. http://dx.doi.org/10.1021/ef060072x.

4. Wani K.S., Kulkarni A.D. Combined Effect of Pour Point Depressants and Magnetic Field on the Viscosity and Pour Point of Crude Oil. International Journal of Creative Research Thoughts, 2017. P. 260-272.

5. Loskutova Iu.V., Yudina N.V. Antioxidant activity and structural and mechanical properties of oils with polymer additives. Proceedings of the International Conference on the Advanced Materials with Hierarchical Structure for New Technologies and Reliable Structures. Tomsk, October 1-5, 2018. AIP Conference Proceeding. Vol. 2051, P. 020171. https://doi.org/10.1063/1.5083414.

6. Mullakaev M.S., Abramov V.O., Abramova A.V. Ultrasonic automated oil well complex and technology for increasing the productivity of marginal wells. Journal of Petroleum Science and Engineering, 2017. Vol. 159, P. 1-7. http://dx.doi.org/10.1016/j.petrol.2017.09.019.

7. Volkova G.I., Anufriev R.V. Structural and mechanical properties of highly paraffinic crude oil processed in high-frequency acoustic field. Key Engineering Materials, 2016. Vol. 67, P. 55-61. http://dx.doi.org/10.17223/24135542/7/3.

8. Maye P.E.E., Jingyi Y., Taoyan Y., Xinru X. Study on the Modification of Vacuum Residue by Ultrasonic Radiation. China Petroleum Processing and Petrochemical Technology, 2017. Vol. 19(1), P. 114-122.

9. Loskutova Yu.V. Effect of Magnetic Fields on the Paramagnetic, Antioxidant, and Viscous Properties of Oils and Resin-Asphaltene Components. Under Ed. Duncan J.A. Asphaltenes: Characterization, Properties and Applications. Series: Chemical Engineering Methods and Technology. USA: Nova Science Publishers, Jnc., 2010. P. 121-144.

10. Evdokimov I.N., Kornishin K.A. Apparent Disaggregation of Colloids in a Magnetically Treated Crude Oil, Energy \& Fuels, 2009. Vol. 23(8), P. 4016-4020. http://dx.doi.org/10.1021/ef900296e. 